\title{
SUPPLEMENT TO CLASSIFICATION OF THREEFOLD DIVISORIAL CONTRACTIONS
}

\author{
MASAYUKI KAWAKITA
}

\begin{abstract}
Every threefold divisorial contraction to a non-Gorenstein point is a weighted blowup.
\end{abstract}

This supplement finishes the explicit description of a threefold divisorial contraction whose exceptional divisor contracts to a non-Gorenstein point. Contractions to a quotient singularity were treated by Kawamata [8]. The author's study (see [7]), based on the singular Riemann-Roch formula, provided the classification except for the case of small discrepancies. On the other hand, Hayakawa (see [1], [2], [3]) classified those with discrepancy at most 1 by the fact that there exists only a finite number of divisors with such discrepancies over a fixed singularity. The only case left was when it is a contraction to a $c D / 2$ point with discrepancy 2 . We demonstrate its classification in Theorem 2 by the method in [7]. It turns out that every contraction is a weighted blowup.

Theorem 1. Let $f: Y \rightarrow X$ be a threefold divisorial contraction whose exceptional divisor $E$ contracts to a non-Gorenstein point $P$. Then $f$ is a weighted blowup of the singularity $P \in X$ embedded into a cyclic quotient of a smooth fivefold.

Our method of classification is to study the structure of the bigraded ring $\bigoplus_{i, j} f_{*} \mathcal{O}_{Y}\left(i K_{Y}+j E\right) / f_{*} \mathcal{O}_{Y}\left(i K_{Y}+j E-E\right)$. We find local coordinates at $P$ to meet this structure, and we verify that $f$ should be a certain weighted blowup. The choice of local coordinates is restricted by the action of the cyclic group, which makes easier the classification in the non-Gorenstein case. We do not know if this method is sufficient to settle all the remaining Gorenstein cases in [4], [5], and [6] with discrepancy at most 4.

Received April 13, 2011. Revised August 17, 2011. Accepted September 4, 2011. 2010 Mathematics Subject Classification. Primary 14E30; Secondary 14J30.

Author's work partially supported by Japan Society for the Promotion of Science, Grant-in-Aid for Young Scientists (A) 20684002. 
By a threefold divisorial contraction to a point, we mean a projective morphism $f:(Y \supset E) \rightarrow(X \ni P)$ between terminal threefolds such that $-K_{Y}$ is $f$-ample and the exceptional locus $E$ is a prime divisor contracting to a point $P$. We will treat $f$ on the germ at $P$ in the complex analytic category. According to $[7$, Theorems $1.2,1.3]$, the only case left is

$$
\text { type e1 with } P=\mathrm{c} D / 2 \text {, discrepancy } a / n=4 / 2
$$

in $[7$, Table 3]. We will prove the following theorem.

THEOREM 2. Suppose that $f$ is a divisorial contraction of type e1 to a $c D / 2$ point with discrepancy 2 . Then $f$ is the weighted blowup with $\operatorname{wt}\left(x_{1}, x_{2}\right.$, $\left.x_{3}, x_{4}, x_{5}\right)=((r+1) / 2,(r-1) / 2,2,1, r)$ with $r \geq 7, r \equiv \pm 1 \bmod 8$ for a suitable identification

$$
P \in X \simeq o \in\left(\begin{array}{c}
x_{1}^{2}+x_{4} x_{5}+p\left(x_{2}, x_{3}, x_{4}\right)=0 \\
x_{2}^{2}+q\left(x_{1}, x_{3}, x_{4}\right)+x_{5}=0
\end{array}\right) \subset \mathbb{C}_{x_{1} x_{2} x_{3} x_{4} x_{5}}^{5} / \frac{1}{2}(1,1,1,0,0),
$$

such that $p$ is of weighted order more than $r$ and $q$ is weighted homogeneous of weight $r-1$ for the weights distributed above.

The proof is along the argument in [7, Section 7]. Henceforth, $f:(Y \supset$ $E) \rightarrow(X \ni P)$ is a divisorial contraction of type e1 to a $\mathrm{c} D / 2$ point with discrepancy 2. By [7, Table 3], $Y$ has only one singular point, say, $Q$, at which $E$ is not Cartier. $Q$ is a quotient singularity of type $(1 / 2 r)(1,-1, r+4)$ with $r \geq 7, r \equiv \pm 1 \bmod 8$.

We set vector spaces $V_{i}=V_{i}^{0} \oplus V_{i}^{1}$ with

$$
\begin{aligned}
V_{i}^{0} & :=f_{*} \mathcal{O}_{Y}(-i E) / f_{*} \mathcal{O}_{Y}(-(i+1) E), \\
V_{i}^{1} & :=f_{*} \mathcal{O}_{Y}\left(K_{Y}-(i+2) E\right) / f_{*} \mathcal{O}_{Y}\left(K_{Y}-(i+3) E\right) .
\end{aligned}
$$

They are zero for negative $i$, and we have the bigraded ring $\bigoplus V_{i}$ by a local isomorphism $\mathcal{O}_{X}\left(2 K_{X}\right) \simeq \mathcal{O}_{X}$. To study its structure in the lower-degree part, we first compute the dimensions of $V_{i}^{j}$ in terms of the finite sets

$N_{i}:=\left\{\left(l_{1}, l_{2}, l_{3}, l_{4}, l_{5}\right) \in \mathbb{Z}_{\geq 0}^{5} \mid \frac{r+1}{2} l_{1}+\frac{r-1}{2} l_{2}+2 l_{3}+l_{4}+r l_{5}=i, l_{1}, l_{2} \leq 1\right\}$.

$N_{i}$ is decomposed into $N_{i}^{0} \sqcup N_{i}^{1}$ with $N_{i}^{j}:=\left\{\left(l_{1}, l_{2}, l_{3}, l_{4}, l_{5}\right) \in N_{i} \mid l_{1}+l_{2}+\right.$ $\left.l_{3} \equiv j \bmod 2\right\}$. 
Lemma 3. We have $\operatorname{dim} V_{i}^{j}=\# N_{i}^{j}$.

Proof. We follow the notation in [7]. We have $\left(r_{Q}, b_{Q}, v_{Q}\right)=(2 r, r+4,2)$ and $E^{3}=1 / r$ by [7, Tables 2,3]. By $\operatorname{dim} V_{i}^{j}=d(j,-i-2 j)$ for $i \geq-2$ in [7, (2.8)], the equality in $[7,(2.6)]$ for $(j,-i-2 j)$ implies that for $i \geq 0$,

$$
\operatorname{dim} V_{i}^{j}-\operatorname{dim} V_{i-2}^{1-j}=\frac{2 i+1}{r}+B_{2 r}(2 i+r j+2)-B_{2 r}(2 i+r j) .
$$

Here $B_{2 r}(k)=(\bar{k} \cdot \overline{2 r-k}) / 2 r$, and ${ }^{-}$denotes the residue modulo $2 r$. On the other hand, by $N_{i}^{j}=\left(N_{i-2}^{1-j}+(0,0,1,0,0)\right) \sqcup\left\{\left(l_{1}, l_{2}, 0, l_{4}, l_{5}\right) \in N_{i}^{j}\right\}$,

$$
\begin{aligned}
& \# N_{i}^{j}-\# N_{i-2}^{1-j} \\
& \quad= \begin{cases}\#\left\{\left(0,0,0, l_{4}, l_{5}\right) \in N_{i}^{0}\right\}+\#\left\{\left(1,1,0, l_{4}, l_{5}\right) \in N_{i}^{0}\right\} & \text { for } j=0, \\
\#\left\{\left(0,1,0, l_{4}, l_{5}\right) \in N_{i}^{1}\right\}+\#\left\{\left(1,0,0, l_{4}, l_{5}\right) \in N_{i}^{1}\right\} & \text { for } j=1 .\end{cases}
\end{aligned}
$$

The lemma follows by verifying the coincidence of their right-hand sides.

We will find bases of $V_{i}$ starting with an arbitrary identification

$$
P \in X \simeq o \in(\phi=0) \subset \mathbb{C}_{x_{1} x_{2} x_{3} x_{4}}^{4} / \frac{1}{2}(1,1,1,0) .
$$

For a semi-invariant function $h, \operatorname{ord}_{E} h$ denotes the order of $h$ along $E$.

\section{LEMMA 4.}

(i) We have $\operatorname{ord}_{E} x_{4}=1$ and $\operatorname{ord}_{E} x_{i} \geq 2$ for $i=1,2,3$. There exists some $k$ with $\operatorname{ord}_{E} x_{k}=2$. We set $x_{k}=x_{3}$ by permutation.

(ii) For $i<(r-1) / 2$, the monomials $x_{3}^{l_{3}} x_{4}^{l_{4}}$ for $\left(0,0, l_{3}, l_{4}, 0\right) \in N_{i}$ form a basis of $V_{i}$. In particular, for $k=1,2$, $\operatorname{ord}_{E} \bar{x}_{k} \geq(r-1) / 2$ for $\bar{x}_{k}:=$ $x_{k}+\sum c_{k l_{3} l_{4}} x_{3}^{l_{3}} x_{4}^{l_{4}}$ with some $c_{k l_{3} l_{4}} \in \mathbb{C}$, with summation over $\left(0,0, l_{3}\right.$, $\left.l_{4}, 0\right) \in \bigcup_{i<(r-1) / 2} N_{i}^{1}$.

(iii) There exists some $k$ with $\operatorname{ord}_{E} \bar{x}_{k}=(r-1) / 2$ such that the monomials $\bar{x}_{k}$ and $x_{3}^{l_{3}} x_{4}^{l_{4}}$ for $\left(0,0, l_{3}, l_{4}, 0\right) \in N_{(r-1) / 2}$ form a basis of $V_{(r-1) / 2}$. We set $\bar{x}_{k}=\bar{x}_{2}$ by permutation; then $\operatorname{ord}_{E} \hat{x}_{1} \geq(r+1) / 2$ for $\hat{x}_{1}:=\bar{x}_{1}+$ $\sum c_{l_{2} l_{3} l_{4}} \bar{x}_{2}^{l_{2}} x_{3}^{l_{3}} x_{4}^{l_{4}}$ with some $c_{l_{2} l_{3} l_{4}} \in \mathbb{C}$, with summation over $\left(0, l_{2}, l_{3}\right.$, $\left.l_{4}, 0\right) \in N_{(r-1) / 2}^{1}$.

(iv) We have $\operatorname{ord}_{E} \hat{x}_{1}=(r+1) / 2$. For $i<r-1$, the monomials $\hat{x}_{1}^{l_{1}} \bar{x}_{2}^{l_{2}} x_{3}^{l_{3}} x_{4}^{l_{4}}$ for $\left(l_{1}, l_{2}, l_{3}, l_{4}, 0\right) \in N_{i}$ form a basis of $V_{i}$. 
(v) Set $\tilde{N}_{i}:=\left\{\left(l_{1}, l_{2}, l_{3}, l_{4}, l_{5}\right) \in \mathbb{Z}_{\geq 0}^{5} \mid((r+1) / 2) l_{1}+((r-1) / 2) l_{2}+2 l_{3}+l_{4}+\right.$ $\left.r l_{5}=i\right\}$, and set $\tilde{N}_{i}^{0}:=\left\{\left(l_{1}, l_{2}, l_{3}, l_{4}, l_{5}\right) \in \tilde{N}_{i} \mid l_{1}+l_{2}+l_{3}\right.$ even $\}$. The monomials $\hat{x}_{1}^{l_{1}} \bar{x}_{2}^{l_{2}} x_{3}^{l_{3}} x_{4}^{l_{4}}$ for $\left(l_{1}, l_{2}, l_{3}, l_{4}, 0\right) \in \tilde{N}_{r-1}^{0}$ have one nontrivial relation, say, $\psi$, in $V_{r-1}^{0}$. The natural exact sequence below is exact.

$$
0 \rightarrow \mathbb{C} \psi \rightarrow \bigoplus_{\left(l_{1}, l_{2}, l_{3}, l_{4}, 0\right) \in \tilde{N}_{r-1}} \mathbb{C} \hat{x}_{1}^{l_{1}} \bar{x}_{2}^{l_{2}} x_{3}^{l_{3}} x_{4}^{l_{4}} \rightarrow V_{r-1} \rightarrow 0
$$

(vi) We have $\operatorname{ord}_{E} \psi=r$. The natural exact sequence below is exact.

$$
0 \rightarrow \mathbb{C} x_{4} \psi \rightarrow \bigoplus_{\left(l_{1}, l_{2}, l_{3}, l_{4}, l_{5}\right) \in \tilde{N}_{r}} \mathbb{C} \hat{x}_{1}^{l_{1}} \bar{x}_{2}^{l_{2}} x_{3}^{l_{3}} x_{4}^{l_{4}} \psi^{l_{5}} \rightarrow V_{r} \rightarrow 0 .
$$

Proof. We follow the proof of [7, Lemma 7.2], using the computation of Lemma 3. Claim (i) above follows from $\operatorname{dim} V_{1}^{0}=1, \operatorname{dim} V_{1}^{1}=0$, and $\operatorname{dim} V_{2}^{1}=1$. Then $V_{4}^{0}$ is spanned by $x_{3}^{2}$ and $x_{4}^{4}$, which should form a basis of $V_{4}^{0}$ by $\operatorname{dim} V_{4}^{0}=2$. Now (ii)-(v) follow from the same argument as in [7, Lemma 7.2]. We obtain the sequence in (vi) also, which is exact possibly except for the middle. Its exactness is verified by comparing dimensions. $\square$

Corollary 5. We distribute weights $\operatorname{wt}\left(\hat{x}_{1}, \bar{x}_{2}, x_{3}, x_{4}\right)=((r+1) / 2$, $(r-1) / 2,2,1)$ to the coordinates $\hat{x}_{1}, \bar{x}_{2}, x_{3}, x_{4}$ obtained in Lemma 4 . Then $\phi$ in (1) is of form

$$
\phi=c x_{4} \psi+\phi_{>r}\left(\hat{x}_{1}, \bar{x}_{2}, x_{3}, x_{4}\right)
$$

with $c \in \mathbb{C}$ and a function $\phi_{>r}$ of weighted order more than $r$, where $\psi$ is as in Lemma 4(v).

Proof. Decompose $\phi=\phi_{\leq r}+\phi_{>r}$ into the part $\phi_{\leq r}$ of weighted order at most $r$ and $\phi_{>r}$ of weighted order more than $r$. Then $\operatorname{ord}_{E} \phi_{\leq r}=\operatorname{ord}_{E} \phi_{>r}>$ $r$, so $\phi_{\leq r}$ is mapped to zero by the natural homomorphism

$$
\bigoplus_{\left(l_{1}, l_{2}, l_{3}, l_{4}, 0\right) \in \bigcup_{i \leq r} \tilde{N}_{i}^{0}} \mathbb{C} \hat{x}_{1}^{l_{1}} \bar{x}_{2}^{l_{2}} x_{3}^{l_{3}} x_{4}^{l_{4}} \rightarrow \mathcal{O}_{X} / f_{*} \mathcal{O}_{Y}(-(r+1) E),
$$

whose kernel is $\mathbb{C} x_{4} \psi$ by Lemma $4(\mathrm{iv})-(\mathrm{vi})$.

We will derive an expression of the germ $P \in X$ in Theorem 2. By [9, Remark 23.1], the c $D / 2$ point $P \in X$ has an identification in (1) with $\phi$ either of

$$
\phi=x_{1}^{2}+x_{2} x_{3} x_{4}+x_{2}^{2 \alpha}+x_{3}^{2 \beta}+x_{4}^{\gamma}
$$


or of

$$
\phi=x_{1}^{2}+x_{2}^{2} x_{4}+\lambda x_{2} x_{3}^{2 \alpha-1}+g\left(x_{3}^{2}, x_{4}\right),
$$

with $\alpha, \beta \geq 2, \gamma \geq 3, \lambda \in \mathbb{C}$, and $g \in\left(x_{3}^{4}, x_{3}^{2} x_{4}^{2}, x_{4}^{3}\right)$. As its general elephant has type $D_{k}$ with $k \geq 2 r$ by [7, Lemma 5.2(i)], we have

$$
\gamma \geq r \quad \text { in }(\mathrm{A}), \quad \text { ord } g\left(0, x_{4}\right) \geq r \quad \text { in }(\mathrm{B}) .
$$

Lemma 6. Case (A) does not happen.

Proof. By Lemma 4(i), $\operatorname{ord}_{E} x_{4}=1, \operatorname{ord}_{E} x_{i} \geq 2$ for $i=1,2,3$, and some $\operatorname{ord}_{E} x_{i}=2$. We have ord $x_{1} \geq 3$ by the relation $-x_{1}^{2}=x_{2} x_{3} x_{4}+x_{2}^{2 \alpha}+x_{3}^{2 \beta}+$ $x_{4}^{\gamma}$ and (2). Thus, we may set $\operatorname{ord}_{E} x_{3}=2$ by permutation and construct $\bar{x}_{1}, \bar{x}_{2}$ as in Lemma $4(\mathrm{ii})$.

Let $W_{(r-1) / 2}$ be the subspace of $V_{(r-1) / 2}$ spanned by the monomials in $x_{3}, x_{4}$. If $\bar{x}_{1} \notin W_{(r-1) / 2}$, the triple $\left(\bar{x}_{1}, x_{3}, x_{4}\right)$ plays the role of $\left(\bar{x}_{2}, x_{3}, x_{4}\right)$ in Lemma 4(iii). We construct $\hat{x}_{2}$ as in Lemma 4(iii) to obtain a quartuple $\left(\hat{x}_{2}, \bar{x}_{1}, x_{3}, x_{4}\right)$, and distribute $\operatorname{wt}\left(\hat{x}_{2}, \bar{x}_{1}, x_{3}, x_{4}\right)=((r+1) / 2,(r-1) / 2,2,1)$ as in Corollary 5. Set $\bar{x}_{1}=x_{1}+p_{1}\left(x_{3}, x_{4}\right)$ and $\hat{x}_{2}=x_{2}+p_{2}\left(\bar{x}_{1}, x_{3}, x_{4}\right)$, and rewrite $\phi$ as

$$
\phi=\left(\bar{x}_{1}-p_{1}\right)^{2}+\left(\hat{x}_{2}-p_{2}\right) x_{3} x_{4}+\left(\hat{x}_{2}-p_{2}\right)^{2 \alpha}+x_{3}^{2 \beta}+x_{4}^{\gamma} .
$$

Here, $\phi$ has the term $\bar{x}_{1}^{2}$ of weight $r-1$, which contradicts Corollary 5 .

Hence, $\bar{x}_{1} \in W_{(r-1) / 2}$, and we obtain a quartuple $\left(\hat{x}_{1}, \bar{x}_{2}, x_{3}, x_{4}\right)$ by $\hat{x}_{1}=$ $x_{1}+p_{1}\left(x_{3}, x_{4}\right), \bar{x}_{2}=x_{2}+p_{2}\left(x_{3}, x_{4}\right)$ as in Lemma 4 . Distribute $\operatorname{wt}\left(\hat{x}_{1}, \bar{x}_{2}, x_{3}\right.$, $\left.x_{4}\right)=((r+1) / 2,(r-1) / 2,2,1)$, and rewrite $\phi$ as

$$
\phi=\left(\hat{x}_{1}-p_{1}\right)^{2}+\left(\bar{x}_{2}-p_{2}\right) x_{3} x_{4}+\left(\bar{x}_{2}-p_{2}\right)^{2 \alpha}+x_{3}^{2 \beta}+x_{4}^{\gamma}
$$

So $\phi$ has the term $\bar{x}_{2} x_{3} x_{4}$ of weight $(r+5) / 2$, whence $(r+5) / 2 \geq r$ by Corollary 5 , a contradiction to $r \geq 7$.

Lemma 7. The germ $P \in X$ has an expression in Theorem 2, with $q$ not of form $\left(x_{3} s\left(x_{3}^{2}, x_{4}\right)\right)^{2}$, such that each $\operatorname{ord}_{E} x_{i}$ coincides with wt $x_{i}$ distributed in Theorem 2.

Proof. We have case (B) by Lemma 6. We have $\operatorname{ord}_{E} x_{4}=1$ and $\operatorname{ord}_{E} x_{1} \geq$ 3 as in (A), so $\operatorname{ord}_{E} x_{2} \geq 3$ and $\operatorname{ord}_{E} x_{3}=2$. We construct $\bar{x}_{1}, \bar{x}_{2}$ as in Lemma 4(ii). By the same reason as in the proof of Lemma 6 , we obtain 
$\bar{x}_{1} \in W_{(r-1) / 2}$ and a quartuple $\left(\hat{x}_{1}, \bar{x}_{2}, x_{3}, x_{4}\right)$ by $\hat{x}_{1}=x_{1}+p_{1}\left(x_{3}, x_{4}\right), \bar{x}_{2}=$ $x_{2}+p_{2}\left(x_{3}, x_{4}\right)$. Distribute wt $\left(\hat{x}_{1}, \bar{x}_{2}, x_{3}, x_{4}\right)=((r+1) / 2,(r-1) / 2,2,1)$, and rewrite $\phi$ as

$$
\phi=\left(\hat{x}_{1}-p_{1}\right)^{2}+\left(\bar{x}_{2}-p_{2}\right)^{2} x_{4}+\lambda\left(\bar{x}_{2}-p_{2}\right) x_{3}^{2 \alpha-1}+g\left(x_{3}^{2}, x_{4}\right) .
$$

So $\phi$ has the term $\bar{x}_{2}^{2} x_{4}$ of weight $r$ and should be of form

$$
\phi=\left(\bar{x}_{2}^{2}+h\left(\hat{x}_{1}, \bar{x}_{2}, x_{3}, x_{4}\right)\right) x_{4}+\phi_{>r}\left(\hat{x}_{1}, \bar{x}_{2}, x_{3}, x_{4}\right)
$$

as in Corollary 5 with $\psi=\bar{x}_{2}^{2}+h\left(\hat{x}_{1}, \bar{x}_{2}, x_{3}, x_{4}\right)$. In particular, $p_{2}=0$, as otherwise $p_{2} \bar{x}_{2} x_{4}$ would be of weighted order less than $r$, and one can write

$$
\phi=\hat{x}_{1}^{2}+x_{4} \psi+p\left(\bar{x}_{2}, x_{3}, x_{4}\right), \quad \psi=\bar{x}_{2}^{2}+q\left(\hat{x}_{1}, x_{3}, x_{4}\right),
$$

where $p$ is of weighted order more than $r$ and $q$ is weighted homogeneous of weight $r-1$. A desired expression is derived by setting $x_{5}:=-\psi$ and replacing $x_{4}$ with $-x_{4}$. Thus $q$ is not of form $\left(x_{3} s\left(x_{3}^{2}, x_{4}\right)\right)^{2}$ by Lemma 4 (iii) and $\operatorname{ord}_{E}\left(\bar{x}_{2}^{2}+q\right)=r$.

Take an expression of the germ $P \in X$ in Theorem 2 by Lemma 7 . We apply the extension of [7, Lemma 6.1] to the case when $X$ is embedded into a cyclic quotient of $\mathbb{C}^{5}$. Let $g: Z \rightarrow X$ be the weighted blowup with wt $x_{i}=$ $\operatorname{ord}_{E} x_{i}$. By direct calculation, we verify the assumptions of [7, Lemma 6.1] and that $Z$ is smooth outside the strict transform of $x_{1} x_{2} x_{3} x_{4} x_{5}=0$. We need the condition $q \neq\left(x_{3} s\right)^{2}$ to check that the restriction $\bar{F} \cap Z$ of the exceptional locus in the ambient space defines an irreducible reduced 2cycle on $Z$. Therefore, $f$ should coincide with $g$ by [7, Lemma 6.1], and Theorem 2 is completed.

REMARK 8. Using $H \cap E \simeq \mathbb{P}^{1}$ in the proof of [7, Theorem 5.4], one can show that

(i) if $r \equiv 1 \bmod 8, x_{2} x_{3}^{(r+3) / 4}$ appears in $p$ and $x_{3}^{(r-1) / 2}$ appears in $q$;

(ii) if $r \equiv 7 \bmod 8, x_{3}^{(r+1) / 2}$ appears in $p$ and $x_{1} x_{3}^{(r-3) / 4}$ appears in $q$.

Theorem 1 follows from [1], [2], [3], [7], [8], and Theorem 2.

Acknowledgments. I was motivated to write this supplement by a question of Professor J. A. Chen. He, with Professor T. Hayakawa, informed me that only one case was left. 


\section{REFERENCES}

[1] T. Hayakawa, Blowing ups of 3-dimensional terminal singularities, Publ. Res. Inst. Math. Sci. 35 (1999), 515-570.

[2] - Blowing ups of 3-dimensional terminal singularities, II, Publ. Res. Inst. Math. Sci. 36 (2000), 423-456.

[3] - Divisorial contractions to 3-dimensional terminal singularities with discrepancy one, J. Math. Soc. Japan 57 (2005), 651-668.

[4] M. Kawakita, Divisorial contractions in dimension three which contract divisors to smooth points, Invent. Math. 145 (2001), 105-119.

[5] — Divisorial contractions in dimension three which contract divisors to compound $A_{1}$ points, Compos. Math. 133 (2002), 95-116.

[6] - General elephants of threefold divisorial contractions, J. Amer. Math. Soc. 16 (2003), 331-362.

[7] — , Three-fold divisorial contractions to singularities of higher indices, Duke Math. J. 130 (2005), 57-126.

[8] Y. Kawamata, "Divisorial contractions to 3-dimensional terminal quotient singularities" in Higher-dimensional Complex Varieties (Trento, 1994), de Gruyter, Berlin, 1996, 241-246.

[9] S. Mori, On 3-dimensional terminal singularities, Nagoya Math. J. 98 (1985), 43-66.

Research Institute for Mathematical Sciences

Kyoto University

Kyoto 606-8502

Japan

masayuki@kurims.kyoto-u.ac.jp 